
\title{
25 Research Soure \\ Quantitative Proteomic Analysis of Bi Zhong Xiao Decoction Against Collagen-induced Arthritis Rats in the Early and Late Stages
}

\section{Cailin He}

Xiangya Hospital Central South University

\section{Yang Wang}

Xiangya Hospital Central South University

\section{Yuqi Wen}

Xiangya Hospital Central South University

\section{Teng Li}

Xiangya Hospital Central South University

\section{En Hu}

Xiangya Hospital Central South University

\section{Siqing Zeng}

Xiangya Hospital Central South University

Xingui Xiong ( $\square$ xiongxg07@csu.edu.cn)

Xiangya Hospital Central South University

\section{Research Article}

Keywords: Bi Zhong Xiao decoction, collagen-induced arthritis, proteomics, rheumatoid arthritis, traditional Chinese medicine

Posted Date: December 28th, 2021

DOI: https://doi.org/10.21203/rs.3.rs-1136785/v1

License: (a) (1) This work is licensed under a Creative Commons Attribution 4.0 International License. Read Full License 


\section{Abstract}

Background: Rheumatoid arthritis (RA) is a chronic, progressive, systemic autoimmune inflammatory disease. Bi Zhong Xiao decoction (BZXD) performs multiple functions for rheumatoid arthritis (RA) treatment for decades. In this study, we aimed to study the protein alterations of BZXD in the early and late stages of RA.

Methods: Sprague-Dawley rats were randomly divided into the Control, collagen-induced arthritis (CIA) and BZXD groups. Clinical assessment, paw thickness, weight changes and serum inflammatory cytokine levels were used to evaluate anti-inflammatory effects. Histopathological tests were performed to assess the improvement of inflammation and synovial hyperplasia. Moreover, we analyzed the proteins profiling of synovial tissue samples with different time intervals after BZXD treatment by Isobaric Tag for Relative Absolute (ITRAQ) quantitative proteomics technology. To further explore the interrelationships among differentially expressed proteins (DEPs), we used DAVID Bioinformatics Resources v6.8 and STRING 11.0 for bioinformatics analysis. Besides, western blot was exerted to verify related proteins.

Results: In our study, BZXD ameliorated joint inflammation, suppressed the pathological changes in arthrosis of CIA rats. The proteomic analysis demonstrated that CIA rats were mainly involved in two significant pathways (the focal adhesion and the ECM-receptor interaction) in the early stage. BZXD down-regulated the expression of proteins involved in these pathways, such as CAV1, CHAD, COL3A1, COL5A2, COL6A1 and COL6A5. Additionally, BZXD exerts anti-inflammatory effects in the late stage mainly by increasing the expression of FASN, and affecting fatty acid metabolism.

Conclusion: BZXD exerts therapeutic effects on RA through multi-pathways in the early and late stages. This work may provide proteomic clues for treating RA by BZXD.

\section{Introduction}

Rheumatoid arthritis (RA) is a chronic, progressive, systemic autoimmune inflammatory disease.(1) Joint swelling and synovial inflammation are the dominant features in the early stage of RA,(2) and continuous progression can lead to significant cartilage loss and bone erosion. $(3,4)$ If not treated timely and adequately, the disease can lead to joint deformities and loss of function.(5) Traditional disease-modifying antirheumatic drugs (DMARDs), biological agents or JAK inhibitors are critical in preventing RA but may have potentially adverse events.(6) Furthermore, these drugs are costly, and not all patients respond well to them.(7) Therefore, finding a safe, effective and cheap medicine has become an inevitable trend of RA research.

Chinese herbal medicine, the main part of traditional Chinese medicine (TCM), has been widely used in the therapy of RA and related disorders, or called "Bi syndrome", with promising outcomes from the ancient past of China.(8) In TCM theories, herbal formulations were administrated based on the identification of Chinese medicine patterns in the patient by analyzing the symptoms and characteristics, which emphasize individual treatment.(9) Herbal formulations have been reported to play important role in anti-inflammatory and anti-arthritic activity by influencing multiple pathways involved in the disease process. $(10,11)$ It is also considered valuable in treating RA because it can greatly improve the quality of life, prevent joint structural damage, and is highly tolerated by RA patients. $(12,13)$ Bi Zhong Xiao decoction (BZXD), a traditional Chinese herbal formula, has been used clinically for many years in our department to treat RA and has no significant side effects. In our previous studies, it was found that BZXD can improve the active movement of the joint and the ability of daily life in RA patients. $(14,15)$ It also decreases the degree of clinical symptoms of arthritis, paw volume, arthritic score, inhibits the inflammatory cytokines and the 
formation of synovial pannus, reduces the degradation of cartilage matrix in collagen-induced arthritis (CIA) rats.(16, 17) Also we investigated the mechanisms by which the three important components of BZXD, paeoniflorin, ferulic acid, and coumaric acid inhibit inflammation and bone erosion in CIA rats.(18-21) However, the dynamic protein network related to the pathogenesis of the CIA rats at different developmental stages and the overall therapeutic mechanism of BZXD remains unknown.

Due to the complexity of systematic pathologies of RA, the exact pathogenesis mechanism of RA has not been fully understood. On the other hand, TCM is rather complex both in the effective chemical components and drug action mechanisms. Recent advances in proteomics provide a new opportunity to reveal the pathological mechanism of RA and the targeted proteins and networks related to drug actions. Especially, the development of the quantitative proteomic technique of isobaric tags for relative and absolute quantification (iTRAQ) with strong cation exchange (SCX)-reverse-phase liquid chromatography-tandem mass spectrometry (SCX-RPLC-MS/MS), which would be the more powerful proteomics methodologies in the pathogenesis mechanism and drug action of RA research.(22-24)

To discover the targeted proteins and predict proteomic networks of BZXD anti-arthritic effect in CIA rats, iTRAQbased quantitative proteomics was used in the present study to identify differentially expressed proteins (DEPs) in synovial tissue at different time points. Bioinformatics analysis was used to dynamically analyze the biological functions, pathways and interaction network of these DEPs. Furthermore, a major DEP was selectively validated by western blot.

\section{Material And Methods}

\section{Preparation of BZXD}

BZXD consists of 9 herbs: Baihuasheshecao, Zhongjiefeng, Tengligen, Yiyiren, Danshen, Luoshiteng, Baishao, Fengxiantougucao and Gancao at a dry-weight ratio of 30:30:30:30:15:15:15:10:5 (Table 1). The herbs were purchased from Central South University Xiangya Hospital pharmacy and the quality was agreed with the People's Republic of China Pharmacopoeia (2015). They were authenticated by Prof. Suiyu Hu, Department of Chinese herbal medicine of Central South University (Changsha, China) and voucher specimens were deposited at the authors' laboratory. We calculated the human daily herbal dose and converted it to a rat daily dose using the body surface area of an individual weighing $70 \mathrm{~kg}$ as previously described;(17) the daily dose per rat was thus $16.2 \mathrm{~g} / \mathrm{kg}$ (crude drug/weight). These crude drugs were processed as follows: mixed, soaked for 1 hour, and boiled twice in a multifunctional extraction tank (the amount of water added was 8 times and 10 times of crude drug, respectively, boiled for 1.5 hours and 1 hour, respectively). Combined the two filtrates to make a concentrated decoction, sealed and stored in a $4^{\circ} \mathrm{C}$ refrigerator for later use. 
Table 1

Compositions of Bi Zhong Xiao decoction

\begin{tabular}{|c|c|c|c|c|c|}
\hline Plant name & Latin name & Chinese name & $\begin{array}{l}\text { Medicinal } \\
\text { part }\end{array}$ & Ratio & $\begin{array}{l}\text { Specimen } \\
\text { number }\end{array}$ \\
\hline $\begin{array}{l}\text { Hedyotis diffusa } \\
\text { Willd. }\end{array}$ & Hedyotis Diffusae Herba & Baihuasheshecao & $\begin{array}{l}\text { Whole } \\
\text { Herb }\end{array}$ & 30 & 20070306 \\
\hline $\begin{array}{l}\text { Sarcandra } \\
\text { glabra(Thunb.)Nakai. }\end{array}$ & Herba Sarcandrae & Zhongjiefeng & $\begin{array}{l}\text { Whole } \\
\text { Herb }\end{array}$ & 30 & 19062901 \\
\hline $\begin{array}{l}\text { Actinidia arguta } \\
\text { (Sieb. et Zucc) } \\
\text { Planch. ex Miq. }\end{array}$ & Actinidia Chinensis Planch & Tengligen & Root & 30 & 19010314 \\
\hline $\begin{array}{l}\text { Coix lacryma-jobi } \\
\text { L.var.mayuen } \\
\text { (Roman.) Stapf. }\end{array}$ & Coicis Semen & Yiyiren & Seed & 30 & 20072307 \\
\hline $\begin{array}{l}\text { Salvia miltiorrhiza } \\
\text { Bunge. }\end{array}$ & Radix Salviae Miltiorrhizae & Danshen & Root & 15 & 20080410 \\
\hline $\begin{array}{l}\text { Trachelospermum } \\
\text { jasminoides (Lindl.) } \\
\text { Lem. }\end{array}$ & Trachelospermumjasminoides & Luoshiteng & $\begin{array}{l}\text { Leaf and } \\
\text { Stem }\end{array}$ & 15 & 19080501 \\
\hline $\begin{array}{l}\text { Paeonia lactiflora } \\
\text { Pall. }\end{array}$ & Paeoniae Radix Alba & Baishao & Root & 15 & 20041711 \\
\hline $\begin{array}{l}\text { Speranskia } \\
\text { tuberculate (Bunge) } \\
\text { Baill. }\end{array}$ & Impatiens Balsamina & Fengxiantougucao & $\begin{array}{l}\text { Whole } \\
\text { Herb }\end{array}$ & 10 & 19091208 \\
\hline $\begin{array}{l}\text { Glycyrrhiza uralensis } \\
\text { Fisch. }\end{array}$ & Radix Glycyrrhizae & Gancao & Root & 5 & 20040108 \\
\hline
\end{tabular}

\section{Animals and collagen-induced arthritis (CIA) model}

Adult specific-pathogen-free Sprague Dawley rats of 6-7 weeks old, weighing 180 to $200 \mathrm{~g}$, male and female each half, were provided by the Laboratory Animal Centre of Central South University (Changsha, China). All rats were housed in a well-ventilated room at $25^{\circ} \mathrm{C}$, with a $12 \mathrm{~h}$ dark/light cycle with free access to water and food for 1 week to adapt to the environment. All experimental protocols were approved by the Animal Ethics Committee of Central South University (No: 2020sydw0898) and were implemented in accordance with the Guide for the Care and Use of Laboratory Animals of the National Institutes of Health (NIH Publication No. 85-23, revised 1996).

According to the Protocol For the Successful Induction of Collagen-Induced Arthritis (CIA) in Rats, Bovine type II collagen (B囚C, 2mg/ml, Chondrex, Inc, Washington DC, USA) completely mixed with acetic acid and then mixed fully with complete Freund's adjuvant (CFA, 1mg/ml, Sigma-Aldrich Co., St Louis, MO, USA). On day 0, rats were injected intradermally at the base of the tail with $200 \mu \mathrm{g}$ collagen/CFA emulsion for primary immunization. On day 7, rats were given $100 \mu \mathrm{g}$ of collagen/incomplete Freund's adjuvant (IFA, 1 mg/ml, Sigma-Aldrich) emulsion for secondary immunization in the same way.

Rats were randomly assigned to 3 groups ( $n=10$ per day per group): Control, CIA and BZXD group. At 14 days after the primary immunization, rats in the BZXD group were administrated BZXD once a day at a dose of $16.2 \mathrm{~g} / \mathrm{kg}$ for 28 days; rats in the Control and CIA groups were treated with an equal volume of saline solution. 


\section{Arthritis assessments}

Clinical assessment, paw thickness, weight, serum inflammatory cytokine levels and histologic assessment were evaluated after arthritis onset. The incidence and severity of arthritis were evaluated using the clinical scoring system. The severity of arthritis in the foot was scored on a scale of 0-3,(25) where 0 : no joint swelling; 1 : mild joint swelling; 2: moderate joint swelling; 3: severe joint swelling (Figure 1E). The disease score of the joints was calculated for each animal (maximum score 12 per rat). At least one entire foot was swollen, including the toes, to be considered as a successful arthritis induction. The thickness of the right paw of rats was measured with compasses and a millimeter ruler in the fixed position after immunization and body weight was monitored throughout the study.

\section{Enzyme-Linked Immunosorbent Assay (ELISA)}

For detection of serum inflammatory cytokine, blood was taken on day 28 and 42 postimmunization, stored at room temperature for 2 hours, centrifuged at $1000 \mathrm{r} / \mathrm{min}$ for 15 minutes. The serum obtained was added to the ELISA kit (specific for rats, Shanghai Zhuocai Biotechnology Co., Ltd., China) to detect the levels of TNF-a and IL-1 $\beta$. The optical densities were detected at $450 \mathrm{~nm}$ by a microplate reader (Rayto RT-6100, Rayto Life and Analytical Sciences Co., Ltd., China). The concentration of TNF- $a$ and IL-1 $\beta$ were calculated with the standards curves.

\section{HE staining}

For histologic assessment, hind paws were removed on day 28 and 42 after immunization, fixed in $10 \%$ neutral formalin for 24 hours, decalcified in the 14\% Ethylene Diamine Tetraacetic Acid (EDTA) decalcify fluid for 5 days, neutralized in $5 \%$ sodium thiosulfate for 3 hours and embedded in dehydrating paraffin. Longitudinal sections (5 - 6 $\mu \mathrm{m}$ ) were cut from the center of the ankle joint, baked in the $60^{\circ} \mathrm{C}$ ovens for 30 minutes and stained with hematoxylin and eosin. Sections were observed by light microscope (CX21; Olympus, Tokyo, Japan) for pathological changes.

\section{Tissue preparation and protein extraction}

The tissues were dissolved in lysis buffer (8 M urea, 4\% chaps, $30 \mathrm{mM}$ HEPES, $1 \mathrm{mM}$ phenylmethanesulfonyl fluoride, $2 \mathrm{mM}$ EDTA and $10 \mathrm{mM}$ DL-Dithiothreitol (DTT)) at $4^{\circ} \mathrm{C}$ for $1 \mathrm{~h}$, ultrasound (water-bath, 5 minutes) and then centrifuged at $20000 \mathrm{rpm}$ for 25 minutes. The supernatants were collected, added DTT, and incubated in a water bath $\left(56^{\circ} \mathrm{C}, 1 \mathrm{~h}\right)$; then, added quickly iodoacetamide to a final concentration of $55 \mathrm{mM}$ and precipitated $\left(-20^{\circ} \mathrm{C}, 3 \mathrm{~h}\right)$ with the addition of precooled acetone, centrifuged $\left(20000 \times \mathrm{g}, 20 \mathrm{~min}, 4^{\circ} \mathrm{C}\right)$. Finally, the proteins were solubilized in $0.5 \mathrm{M}$ triethylammonium bicarbonate plus $0.1 \%$ Sodium dodecyl sulfate and measured their concentration by the Bradford protein assay kit (Ameresco). More details were detailedly performed in our previous study.(20)

\section{ITRAQ method}

Trypsin digestion and iTRAQ labeling were performed according to the manufacturer's protocol (Applied Biosystems). Briefly, $100 \mu \mathrm{g}$ protein of each pooled sample was reduced, alkylated and then digested overnight at $37^{\circ} \mathrm{C}$ with trypsin. Apart ( $\left.1 \mu \mathrm{l}\right)$ of tryptic peptides was taken to detect digestion efficiency using Ultraflex TOF/TOF (Bruker, Germany). The tryptic peptide solution of each sample was labeled with iTRAQ reagents according to the iTRAQ Reagent Multiplex Kit protocol (Applied Biosystem). The tryptic peptide samples were mixed, fractionated and dried as described previously.(20) before further analysis. Identification and quantification of the iTRAQ-labeled samples were performed by Q Exactive LC-MS/MS (Thermo Scientific Co.). To reduce the influence of experimental variation on the proteomics analysis results, three independent MS/MS runs were performed on each sample.(26)

The software used for data acquisition and quantitation was Proteome Discoverer software (Thermo Scientific version 1.3). The data sifted by Proteome Discoverer were used to identify protein using Mascot (version 2.3.0, 
Matrix Science, London, UK) and the Uniprot-rat database (http://www.uniprot.org/). The Mascot search parameters included trypsin, peptides digested with a maximum of one missed cleavage, fixed modification (carbamidomethylation of a cysteine residue), variable modifications (oxidation of methionine GIn-Pyro-Glu of Nterm Q, and iTRAQ 8 plex modification of $\mathrm{N}$ terminal, $\mathrm{K}$ and $\mathrm{Y}$ ), peptide tolerance $15 \mathrm{ppm}$, and the iTRAQ fragment tolerance (0.2 Da). Using these criteria 59,285 spectra were identified with $95 \%$ confidence. The differential expression proteins were identified with the following criteria: $\geq 2$ peptides match, repeatedly identified in three replications, and an averaged ratio-fold change $\geq 1.2$ or $\leq 0.84$ between two groups.

\section{Bioinformatics analyses of DEPs}

Venn diagram and dumbbell charts were exerted to exhibit the DEPs using OriginPro Origin ${ }^{\circledR} 2021$ (version 9.8.0.200) and the ggpubr package in $\mathrm{R}$, respectively. To explore the molecular mechanism, the DEPs were imported into DAVID Bioinformatics Resources v6.8 (https://david.ncifcrf.gov/)(27) to analyze Gene Ontology (GO, which contains a biological process (BP), cellular component (CC) and molecular function (MF)) and Kyoto Encyclopedia of Genes and Genomes (KEGG) pathways. The DEPs were also imported into STRING 11.0 (http://www.string$\mathrm{db} . \mathrm{org} /$ ) to create protein-protein interaction (PPI) networks.

\section{Western blot}

Western blot was performed in synovial tissues (Control d28, CIA d28, BZXD d28, Control d42, CIA d42 and BZXD $\mathrm{d} 42, \mathrm{n}=4)$. Synovial tissues were lysed in RIPA buffer, followed by homogenizing mechanically. The homogenates were centrifuged at $12000 \mathrm{rpm}$ for $15 \mathrm{~min}$ at $4^{\circ} \mathrm{C}$. Protein samples were separated on $11 \%$ sodium dodecyl sulfatepolyacrylamide gel electrophoresis and then transferred onto polyvinylidene difluoride membranes (Bio-Rad).

Following blocking with $5 \%$ skim milk, the transferred membranes were incubated overnight at $4^{\circ} \mathrm{C}$ with rabbit-antirat FASN antibody (10624-1-AP, 1:500; Proteintech, USA) and mouse-anti-rat $\beta$-actin antibody (66009-1-lg, 1:5000; Proteintech, USA). They were subsequently incubated with HRP goat-anti-rabbit IgG (SA00001-2, 1:6000; Proteintech, USA) and HRP goat-anti-mouse IgG (SA00001-1, 1:5000; Proteintech, USA) for 90 min at room temperature. Bands were visualized by electrochemiluminescence detection reagent (Thermo Scientific Pierce) and quantified by densitometry using an Image-Quant image analysis system (Storm Optical Scanner, Molecular Dynamics). The $\beta$ actin was detected simultaneously as a loading control. All western blot analysis for each interested protein was performed in triplicate.

\section{Statistical analysis}

SPSS 26.0 software was used for statistical analysis. The data were expressed as the mean \pm standard deviation (SD). Each time point index difference in the group was analyzed by the analysis of variance of repeated measurement design. Group comparison adopts independent Student's t-test or one-way analysis of variance (ANOVA). A value of $\mathrm{P}<0.05$ was considered statistically significant.

\section{Results}

\section{BZXD treatment ameliorates arthritis in CIA rats}

Day 0 mean the day having the primary immunization and day 7 having booster immunization. On day 14, rats were administrated with BZXD or distilled water. As shown in Fig. 1, compared with the Control group, both CIA and BZXD groups showed a significant increase in the clinical score and paw swelling, whereas a significant weight reduction. As shown in Fig. 1A and 1B, there was no difference in clinical score and paw swelling between groups before day 28. The onset of the significant difference in the clinical score and paw swelling was seen on day 28 . However, the 
most significant weight change was observed on day 21 comparing the CIA versus BZXD group see Fig. 1C. As was expected, IL-1 $\beta$ and TNF-a serum levels were reduced in the BZXD treated animals compared to the CIA rats on day 28 and 42. However, significant increases in the two inflammatory cytokine levels were observed both in the CIA and BZXD groups compared with the Control group (Figure 1D).

HE staining was used to observe the inflammatory cell infiltration in the synovial tissue of the hind ankle joints of rats. As shown in Figure 2, the Control group had no signs of inflammation. Fourteen days after immunization, some inflammatory cell infiltration and synovium hyperplasia were observed in the synovial tissues of rats in the CIA group; a large number of inflammatory cells and bone destruction could be seen in the late stage. On the 28th and 42nd day, the above symptoms in BZXD groups were alleviated compared with the CIA group. These results indicated that BZXD treatment ameliorated inflammation of the joints in CIA rats.

\section{ITRAQ analysis of DEPs}

To define protein alterations changed in disease progression, we performed LC-MS/MS of synovial tissues dissected from all groups. DEPs were screened by the criteria $(\geq 1.2$ or $\leq 0.84)$ as described above. On day 28 , In the $\mathrm{CIA} / \mathrm{Control}$ group, 221 DEPs were identified (120 were up-regulated and 101 were down-regulated); in BZXD/CIA group, 638 DEPs were appraised (299 were up-regulated and 399 were down-regulated) (Fig. 3A). On day 42, 257 DEPs (137 were up-regulated and 120 were down-regulated) and 104 DEPs (56 were up-regulated and 48 were down-regulated) were identified in the CIA/Control group and BZXD/CIA group respectively (Fig. 3C). 146 and 94 overlapping DEPs were identified in both the CIA/Control group and BZXD/CIA group on day 28 and 42, respectively. (Fig. 3B and 3D, Table S1 and S2). Among these DEPs, 94 and 51 proteins were reversely regulated by BZXD treatment.

In addition, 16 overlapping proteins were differentially expressed between the CIA/Control group and BZXD/CIA group at two-time points (day 28 and 42) (Fig. 3E). To visualize their fold change in each individual, we plotted dumbbell charts. Fig. 3F shows that 14 candidate proteins were changed in the CCI group and BZXD group at both time points. On days 28 and 42, there was no difference in GW7_03778 and GW7_15073 proteins between the CCI group and the BZXD group, so they were removed.

\section{Bioinformatics analysis of DEPs Functional classification of DEPs}

To acquire functional information induced by BZXD, we imported 94 and 51 differentially expressed and reversely regulated proteins in DAVID, respectively. GO annotations of the differential proteins (41 biological processes, 15 cellular components and 20 molecular functions) were obtained on day 28. On day 42, we obtained 13 biological processes, 7 cellular components and 6 molecular functions of DEPs. Subsequently, we used the R ggplot2 package to visualize the top $10 \mathrm{GO}$ enrichment terms (Fig. 4A and B).

In the biological process, the proteins were located in negative regulation of JAK-STAT cascade, collagen fibril organization, muscle contraction, cytokine-mediated signaling pathway, and integrin-mediated signaling pathway. For cellular components, DEPs focused on extracellular matrix, extracellular exosome, extracellular space, and proteinaceous extracellular matrix. As for molecular function, DEPs were associated with collagen-binding, protein kinase inhibitor activity, NAD binding, calcium ion binding.

\section{KEGG pathway analysis of DEPs}


To uncover the functional association between the Control group and the BZXD group differential proteins at day 28 and 42, respectively. We conducted a KEGG analysis using DAVID. On day 28, 20 and 58 pathways were enriched at protein expression in the CIA vs Control and BZXD vs CIA comparisons, respectively. 24 and 11 pathways were respectively enriched in the CIA vs Control and BZXD vs CIA comparisons at day 42 . The top 15 significant KEGG pathways were listed in Fig. 5, there were considerable overlaps of pathways between the two groups, suggesting BZXD may ameliorate joint inflammation by regulating these pathways (Table 2). Overall, the top enriched biological pathways for both day 28 and 42 included focal adhesion, carbon metabolism, biosynthesis of antibiotics and metabolic pathways. In addition, the complement and coagulation cascades, ECM-receptor interaction, glycolysis/gluconeogenesis and pyruvate metabolism were enriched significantly on day 28. The KEGG pathway analysis also revealed that the identified DEPs were mainly involved in fatty acid metabolism on day 42. 
Table 2

Overlapping KEGG pathways between the two groups

\begin{tabular}{|c|c|c|c|c|c|}
\hline \multirow[t]{2}{*}{ KEGG Pathways } & \multirow[t]{2}{*}{ Common Genes } & \multicolumn{2}{|c|}{ CIA/Control } & \multicolumn{2}{|c|}{$\mathrm{BZXD} / \mathrm{ClA}$} \\
\hline & & PValue & Count & PValue & Count \\
\hline \multicolumn{6}{|l|}{28 Day } \\
\hline Focal adhesion & $\begin{array}{l}\text { MYLPF, ACTN3, CAV1, COL5A2, COL6A1, CHAD, } \\
\text { TLN2 }\end{array}$ & 0.0011 & 11 & 0.0000 & 39 \\
\hline Carbon metabolism & HK3, PC, ME1, PGP, ENO2 & 0.0021 & 8 & 0.0000 & 33 \\
\hline $\begin{array}{l}\text { Biosynthesis of } \\
\text { antibiotics }\end{array}$ & ENO2 & 0.0051 & 10 & 0.0000 & 40 \\
\hline $\begin{array}{l}\text { ECM-receptor } \\
\text { interaction }\end{array}$ & COL5A2, COL6A1, CHAD & 0.0102 & 6 & 0.0000 & 24 \\
\hline $\begin{array}{l}\text { Glycolysis / } \\
\text { Gluconeogenesis }\end{array}$ & HK3, ALDH2, ENO2 & 0.0199 & 5 & 0.0000 & 14 \\
\hline $\begin{array}{l}\text { Pyruvate } \\
\text { metabolism }\end{array}$ & PC, ALDH2, ME1 & 0.0211 & 4 & 0.0000 & 12 \\
\hline $\begin{array}{l}\text { Complement and } \\
\text { coagulation } \\
\text { cascades }\end{array}$ & CFD, KLKB1, KNG2, KNG1, C2 & 0.0007 & 7 & 0.0000 & 18 \\
\hline Metabolic pathways & $\begin{array}{l}\text { HIBADH, GDA, HEXB, PYGM, NT5C2, ENO2, } \\
\text { PAPSS1, HK3, ALDH2, ME1, GGT5, CKM, ACLY, } \\
\text { PC, FASN, PGP, PLPP3 }\end{array}$ & 0.0113 & 30 & 0.0000 & 96 \\
\hline \multicolumn{6}{|l|}{42 Day } \\
\hline Focal adhesion & MYLPF, COL3A1, ACTN3, COL6A5 & 0.0012 & 12 & 0.0139 & 6 \\
\hline Carbon metabolism & RPIA, PHGDH, GPT & 0.0003 & 10 & 0.0092 & 5 \\
\hline $\begin{array}{l}\text { Biosynthesis of } \\
\text { antibiotics }\end{array}$ & RPIA, PHGDH & 0.0053 & 11 & 0.0620 & 5 \\
\hline $\begin{array}{l}\text { Fatty acid } \\
\text { metabolism }\end{array}$ & FASN, ACADSB & 0.0149 & 5 & 0.0524 & 3 \\
\hline Metabolic pathways & $\begin{array}{l}\text { AHCYL1, GPT, AKR1B8, PAPSS1, ALAD, UROD, } \\
\text { PHGDH, FDPS, ACADSB, RPIA, FASN }\end{array}$ & 0.0046 & 36 & 0.0098 & 17 \\
\hline
\end{tabular}

\section{PPI analysis of DEPs}

We used the STRING database to determine the relationship between 41 DEPs, which were enriched in the above pathways. DEPs with the combined protein interaction score $>0.4$ were selected and used to construct a PPI network. Then, to identify the hub genes of BZXD against CIA, we constructed PPI network by Cytoscape. The hub genes were calculated based on the MNC method by Cytoscape (Table S3). Fig. 6 shows the overall relationships within DEPs.

\section{Validation of DEPs}


Chose FASN with high node degree and important function, and used molecular biotechnology to verify the reliability of quantitative proteomics. This result was consistent with proteomic data. On day 28 and 42, western blot analyses showed that FASN was notedly down-regulated, and BZXD reversed its expressions significantly (Figure7).

\section{Discussion}

In this study, we demonstrated that BZXD could effectively inhibit joint swelling, synovial inflammation, joint destruction and significantly decrease serum pro-inflammatory cytokine (TNF-a and IL- $\beta$ ) levels in CIA rats. The mechanisms of BZXD are complicated because of the complex ingredients of BZXD. Therefore, we conducted a proteomic study on the synovial tissue of CIA rats. Bioinformatic analysis identified several signaling pathways including the metabolic pathways, complement and coagulation cascades, focal adhesion, ECM-receptor interaction, glycolysis/gluconeogenesis, pyruvate metabolism and fatty acid metabolism. We analyzed the DEPs within the above-mentioned signaling pathways and verified a protein with a high node degree and important functions by western blot. Overall, BZXD has played a systemic role, enriching our understanding of the mechanism of treating RA.

ECM-receptor interaction is an important signaling pathway in the progression of RA, which is significantly enriched in the early stage. Extracellular matrices (ECMs) are involved in the pathological process of many genetic and autoimmune diseases and promote disease progression by regulating cell-matrix interactions.(28) ECMs are composed of collagen, elastin, fibronectin, laminins, proteoglycans, hyaluronan, and several glycoproteins such as matricellular proteins. Collagen is the oldest and most abundant component in ECMs, which builds fibers, networks and filaments in ECMs.(29) In our study, PPI analysis showed that multiple collagen subtypes (COL3A1, COL5A2, COL6A1 and COL6A5) are connected closely with high degrees. Among these collagen subtypes, COL3A1 is increased in osteoarthritis cartilage compared to normal cartilage, and its expression may be correlated with the radiographic severity of canine elbow osteoarthritis.(30) Studies have confirmed that IL-1 $\beta$ could induce the upregulated secretion of COL3A1 from the human synoviocyte.(31) Our research demonstrated that BZXD could inhibit the expression of IL-1 $\beta$ and COL3A1 is decreased in the BZXD group relative to the CIA group. Thence, COL3A1 may be a molecular marker for BZXD treating RA. In addition, COL5A2 and COL6A1 were demonstrated as upregulated genes associated with a pathological process of subchondral bone in osteoarthritis. $(32,33)$ These collagens also were upregulated in the CIA group, which indicates that these collagens have implications in the progression of RA. However, BZXD can downregulate the expression of these collagens, which may be the molecular mechanism of BZXD treating RA.

The focal adhesion pathway is a complex and multi-intersection network, which is closely related to the ECM receptor interaction pathway. It can regulate cell movement, proliferation, differentiation, expression and apoptosis. $(34,35)$ The formation of specific adhesion points focal adhesion kinase at the cell membrane-cytoplasm contact is an important step in the cell-matrix interaction.(34) Some studies have shown that the focal adhesion kinase family kinases are overexpressed in RA synovial tissues, which promote synovial fibroblast invasion and migration. $(36,37)$ Thence, the focal adhesion pathway plays an important role in the occurrence, development and progression of RA. This study also suggested that the focal adhesion pathway was significantly enriched in both early and late stages. DEPs (MYLPF, ACTN3, CAV1, CHAD, TLN2, COL3A1, COL5A2, COL6A1 and COL6A5) participated in the focal adhesion pathway. CAV1 is the main component of the caveolae structure, which promotes endocytosis, cell signaling, and endothelial-mediated inflammation.(38) Research has shown that silencing of CAV1 significantly decreased cell proliferation and promoted apoptosis in RA fibroblast-like synoviocytes.(39) Our research shows that BZXD reduces the expression of CAV1 in CIA rats, indicating that CAV1 may be a potential target for BZXD to exert

Page 10/20 
therapeutic effects. In addition, CHAD is mainly localized in the territorial matrix of the deeper parts of the articular cartilage.(40) It mediates intracellular signal transduction between chondrocytes and the ECM through binding to the a2 $\beta 1$ integrin. $(41,42)$ CHAD also binds collagen type II and type VI can inhibit the spreading of chondrocytes. $(43,44)$ CHAD plays a critical role in regulating linkages between collagens and other ECM molecules in vivo, as well as the communication between chondrocytes and their surrounding matrices.(44) In our study, the BZXD group reduced the expression of CHAD compared with the CIA group. Therefore, we speculated that adhesion-related proteins may be an important feature of BZXD in treating RA.

The microenvironment of RA synovial tissue accumulates a variety of cytokines, adipocytokines and metabolic intermediates, which make a variety of metabolic pathways dysregulated, including glycolysis, tricarboxylic acid cycle, pentose phosphate pathway and lipid metabolism. The dysregulation of metabolic pathways deteriorates proinflammatory immune responses and chronic inflammation.(45) Some studies have shown that glycolysis is increased in the RA synovial tissues and leads to persistent synovial inflammation and joint damage.(46-48) In addition, the end product of glycolysis is pyruvate, which could stimulate the expression of vascular endothelial growth factor (VEGF) mRNA.(49) In our previous study,(50) we found that the expression of VEGF was increased in the synovial tissues of CIA rats, while BZXD significantly decreased its level, indicating that BZXD maybe reduce the symptoms of RA by modulating the pyruvate metabolism. This needs further study. In this study, metabolic pathways, pyruvate metabolism, glycolysis/gluconeogenesis were disturbed in the CIA group, while BZXD regulated these disordered metabolic pathways back to normal.

Fatty acid metabolism is a dynamic process of anabolic and catabolic reactions that maintain energy homeostasis. (51) The regulation of fatty acid synthesis is closely related to the physiological and pathophysiological processes regulated by immune cells.(52) Studies have also determined the role of fatty acids in immune response and inflammation, that is, saturated fatty acids promote inflammation, while polyunsaturated fatty acids play an antiinflammatory effect.(53) In our study, KEGG showed that the CIA rat group was significantly enriched in fatty acid metabolism, and BZXD may exert anti-inflammatory effects by regulating fatty acid metabolism. Furthermore, FASN is a key enzyme in the de novo synthesis of lipids. It acts as a central regulator of lipid metabolism and is overexpressed in inflammation and the immune system.(54-56) Interestingly, the previous research(57) reported that FASN is specifically low-expressed in the synovial tissue of CIA rats. This is consistent with our findings. After the pro-inflammatory cytokines TNF-a, adipocytes reduce the secretion of adiponectin, resulting in a decrease in lipid accumulation, thereby inhibiting the expression of FASN in arthritic rats.(57) Our research showed that BZXD can effectively reduce the expression of TNF-a in CIA rats. Meanwhile, compared with the CIA group, the BZXD treatment group increased the expression of FASN. We speculate that BZXD may play a curative effect by regulating the expression of FASN.

\section{Conclusions}

In summary, this proteomics work focuses on an overview of the multiple functions regulated by BZXD for RA treatment. These observations give us a broad understanding of the early and late treatment effects of BZXD on RA.

\section{Declarations}

\section{Acknowledgments}

Not applicable. 


\section{Authors' contributions}

XGX, YW, and CLH conceived and designed the experiments. CLH, YQW, and SQZ performed the experiments. CLH analyzed the data and visualized the figures. CLH drafted the manuscript. XGX, TL, YW, and EH revised it. All authors contributed to the article and approved the submitted version.

\section{Funding}

This work was supported by the National Natural Science Foundation of China (No. 81874407), Hunan Provincial Natural Science Foundation of China (No. 2019JJ40522).

\section{Availability of data and materials}

Data of this study are included in the article and the primary data can be provided from the corresponding author.

\section{Ethics approval and consent to participate}

All experimental protocols in this study involving animals were conducted in accordance with the ethical standards and the international regulations of the usage and welfare of laboratory animals, and were approved by the Animal Ethics Committee of Central South University (No: 2020sydw0898), and carried out in compliance with the ARRIVE guidelines.

\section{Consent for publication}

Not applicable.

\section{Competing interests}

The authors declare that they have no competing interests.

\section{References}

1. Smolen JS, Aletaha D, Barton A, Burmester GR, Emery P, Firestein GS, et al. Rheumatoid arthritis. Nat Rev Dis Primers. 2018;4:18001.

2. Yeo L, Adlard N, Biehl M, Juarez M, Smallie T, Snow M, et al. Expression of chemokines CXCL4 and CXCL7 by synovial macrophages defines an early stage of rheumatoid arthritis. Ann Rheum Dis. 2016;75(4):763-71.

3. Schett G, Gravallese E. Bone erosion in rheumatoid arthritis: mechanisms, diagnosis and treatment. Nat Rev Rheumatol. 2012;8(11):656-64.

4. Weyand CM, Goronzy JJ. Immunometabolism in early and late stages of rheumatoid arthritis. Nat Rev Rheumatol. 2017;13(5):291-301.

5. Sparks JA. Rheumatoid Arthritis. Ann Intern Med. 2019;170(1). 
6. Aletaha D, Smolen JS. Diagnosis and Management of Rheumatoid Arthritis: A Review. JAMA. 2018;320(13):1360-72.

7. Smolen JS, Aletaha D, McInnes IB. Rheumatoid arthritis. Lancet. 2016;388(10055):2023-38.

8. Li X-Z, Zhang S-N. Herbal compounds for rheumatoid arthritis: Literatures review and cheminformatics prediction. Phytother Res. 2020;34(1):51-66.

9. Qiu J. Traditional medicine: a culture in the balance. Nature. 2007;448(7150):126-8.

10. Wang Y, Chen S, Du K, Liang C, Wang S, Owusu Boadi E, et al. Traditional herbal medicine: Therapeutic potential in rheumatoid arthritis. Journal of ethnopharmacology. 2021;279:114368.

11. Xie Y, Mai C-T, Zheng D-C, He Y-F, Feng S-L, Li Y-Z, et al. Wutou decoction ameliorates experimental rheumatoid arthritis via regulating NF-kB and Nrf2: Integrating efficacy-oriented compatibility of traditional Chinese medicine. Phytomedicine. 2021;85:153522.

12. Feng $\mathrm{C}$, Chen $\mathrm{R}$, Wang $\mathrm{K}$, Wen $\mathrm{C}$, Xu Z. Chinese traditional medicine (GuiZhi-ShaoYao-ZhiMu decoction) as an add-on medication to methotrexate for rheumatoid arthritis: a meta-analysis of randomized clinical trials. Ther Adv Chronic Dis. 2021;12:2040622321993438.

13. Wu J, Chen X, Lv Y, Gao K, Liu Z, Zhao Y, et al. Chinese Herbal Formula Huayu-Qiangshen-Tongbi Decoction Compared With Leflunomide in Combination With Methotrexate in Patients With Active Rheumatoid Arthritis: An Open-Label, Randomized, Controlled, Pilot Study. Front Med (Lausanne). 2020;7:484.

14. Liang Q, Tang T, Zhang H. [Clinical investigation of effects of bizhongxiao decoction (BZX) on rheumatoid arthritis on active phase]. Hunan Yi Ke Da Xue Xue Bao. 2000;25(5):449-52.

15. Liang QH, Zhang HX, Tang T. [The effects of bizhongxiao decotion (BZX) on T-lymphocyte subsets in the peripheral blood of patients with rheumatoid arthritis]. Hunan Yi Ke Da Xue Xue Bao. 2001;26(6):534-6.

16. Wang A-Y, Liang Q-H, Luo X, Bao T-C, Li C-Y, Cheng J, et al. Effect of Bizhongxiao Decotion (BZXD) on Some Cytokines in Plasma of Rats with Cll-induced Rheumatoid Arthritis. International journal of biomedical science: IJBS. 2005;1(1):53-6.

17. Guo Y-j, Chen J, Xiong X-g, Wu D, Zhu H, Liang Q-h. Effect of Bizhongxiao decoction and its dismantled formulae on IL-1 and TNF levels in collagen-induced arthritis in rat synovial joints. Theoretical biology \& medical modelling. 2012;9:47.

18. Wu D, Chen J, Zhu H, Xiong X-G, Liang Q-H, Zhang Y, et al. UPLC-PDA determination of paeoniflorin in rat plasma following the oral administration of Radix Paeoniae Alba and its effects on rats with collagen-induced arthritis. Experimental and therapeutic medicine. 2014;7(1):209-17.

19. Zhu H, Liang Q-H, Xiong X-G, Chen J, Wu D, Wang Y, et al. Anti-Inflammatory Effects of the Bioactive Compound Ferulic Acid Contained in Oldenlandia diffusa on Collagen-Induced Arthritis in Rats. Evidence-based complementary and alternative medicine: eCAM. 2014;2014:573801.

20. Yang S, Xing Z, Liu T, Zhou J, Liang Q, Tang T, et al. Synovial tissue quantitative proteomics analysis reveals paeoniflorin decreases LIFR and ASPN proteins in experimental rheumatoid arthritis. Drug Des Devel Ther. 2018;12:463-73.

21. Zhu H, Liang Q-H, Xiong X-G, Wang Y, Zhang Z-H, Sun M-J, et al. Anti-Inflammatory Effects of p-Coumaric Acid, a Natural Compound of, on Arthritis Model Rats. Evidence-based complementary and alternative medicine : eCAM. 2018;2018:5198594.

22. Li R, He Y, Zhu Y, Jiang L, Zhang S, Qin J, et al. Route to Rheumatoid Arthritis by Macrophage-Derived Microvesicle-Coated Nanoparticles. Nano letters. 2019;19(1):124-34. 
23. Chen J, Tang M-S, Xu L-C, Li S, Ge Y, Du J-F, et al. Proteomic analysis of biomarkers predicting the response to triple therapy in patients with rheumatoid arthritis. Biomed Pharmacother. 2019;116:109026.

24. Khanna S, Padhan P, Jaiswal KS, Jain AP, Ghosh A, Tripathy A, et al. Altered mitochondrial proteome and functional dynamics in patients with rheumatoid arthritis. Mitochondrion. 2020;54.

25. Ruth JH, Amin MA, Woods JM, He X, Samuel S, Yi N, et al. Accelerated development of arthritis in mice lacking endothelial selectins. Arthritis research \& therapy. 2005;7(5):R959-R70.

26. Serada S, Fujimoto M, Ogata A, Terabe F, Hirano T, lijima H, et al. iTRAQ-based proteomic identification of leucine-rich alpha-2 glycoprotein as a novel inflammatory biomarker in autoimmune diseases. Ann Rheum Dis. 2010;69(4):770-4.

27. Huang DW, Sherman BT, Lempicki RA. Systematic and integrative analysis of large gene lists using DAVID bioinformatics resources. Nat Protoc. 2009;4(1):44-57.

28. Theocharis AD, Manou D, Karamanos NK. The extracellular matrix as a multitasking player in disease. FEBS J. 2019;286(15):2830-69.

29. Sorushanova A, Delgado LM, Wu Z, Shologu N, Kshirsagar A, Raghunath R, et al. The Collagen Suprafamily: From Biosynthesis to Advanced Biomaterial Development. Adv Mater. 2019;31(1):e1801651.

30. Clements DN, Fitzpatrick N, Carter SD, Day PJR. Cartilage gene expression correlates with radiographic severity of canine elbow osteoarthritis. Vet J. 2009;179(2):211-8.

31. Tang S, Deng S, Guo J, Chen X, Zhang W, Cui Y, et al. Deep Coverage Tissue and Cellular Proteomics Revealed IL$1 \beta$ Can Independently Induce the Secretion of TNF-Associated Proteins from Human Synoviocytes. J Immunol. 2018;200(2):821-33.

32. Guo S-M, Wang J-X, Li J, Xu F-Y, Wei Q, Wang H-M, et al. Identification of gene expression profiles and key genes in subchondral bone of osteoarthritis using weighted gene coexpression network analysis. J Cell Biochem. 2018;119(9):7687-95.

33. Gu H-Y, Yang M, Guo J, Zhang C, Lin L-L, Liu Y, et al. Identification of the Biomarkers and Pathological Process of Osteoarthritis: Weighted Gene Co-expression Network Analysis. Front Physiol. 2019;10:275.

34. Revach O-Y, Grosheva I, Geiger B. Biomechanical regulation of focal adhesion and invadopodia formation. J Cell Sci. 2020;133(20).

35. Paluch EK, Aspalter IM, Sixt M. Focal Adhesion-Independent Cell Migration. Annu Rev Cell Dev Biol. 2016;32:469-90.

36. Shelef MA, Bennin DA, Yasmin N, Warner TF, Ludwig T, Beggs HE, et al. Focal adhesion kinase is required for synovial fibroblast invasion, but not murine inflammatory arthritis. Arthritis research \& therapy. 2014;16(5):464.

37. Shahrara S, Castro-Rueda HP, Haines GK, Koch AE. Differential expression of the FAK family kinases in rheumatoid arthritis and osteoarthritis synovial tissues. Arthritis research \& therapy. 2007;9(5):R112.

38. Codrici E, Albulescu L, Popescu ID, Mihai S, Enciu A-M, Albulescu R, et al. Caveolin-1-Knockout Mouse as a Model of Inflammatory Diseases. J Immunol Res. 2018;2018:2498576.

39. Li S, Jin Z, Lu X. MicroRNA-192 suppresses cell proliferation and induces apoptosis in human rheumatoid arthritis fibroblast-like synoviocytes by downregulating caveolin 1. Mol Cell Biochem. 2017;432(1-2):123-30.

40. Shen Z, Gantcheva S, Mânsson B, Heinegârd D, Sommarin Y. Chondroadherin expression changes in skeletal development. Biochem J. 1998;330 (Pt 1):549-57.

41. Camper L, Heinegârd D, Lundgren-Akerlund E. Integrin alpha2beta1 is a receptor for the cartilage matrix protein chondroadherin. J Cell Biol. 1997;138(5):1159-67.

Page $14 / 20$ 
42. Haglund L, Tillgren V, Addis L, Wenglén C, Recklies A, Heinegård D. Identification and characterization of the integrin alpha2beta1 binding motif in chondroadherin mediating cell attachment. J Biol Chem.

2011;286(5):3925-34.

43. Mansson B, Wenglén C, Mörgelin M, Saxne T, Heinegård D. Association of chondroadherin with collagen type II. J Biol Chem. 2001;276(35):32883-8.

44. Batista MA, Nia HT, Önnerfjord P, Cox KA, Ortiz C, Grodzinsky AJ, et al. Nanomechanical phenotype of chondroadherin-null murine articular cartilage. Matrix Biol. 2014;38:84-90.

45. Pucino V, Certo M, Varricchi G, Marone G, Ursini F, Rossi FW, et al. Metabolic Checkpoints in Rheumatoid Arthritis. Front Physiol. 2020;11:347.

46. Xu D, Liang J, Lin J, Yu C. PKM2: A Potential Regulator of Rheumatoid Arthritis via Glycolytic and Non-Glycolytic Pathways. Front Immunol. 2019;10:2919.

47. Wang C, Xiao Y, Lao M, Wang J, Xu S, Li R, et al. Increased SUMO-activating enzyme SAE1/UBA2 promotes glycolysis and pathogenic behavior of rheumatoid fibroblast-like synoviocytes. JCI Insight. 2020;5(18).

48. Garcia-Carbonell R, Divakaruni AS, Lodi A, Vicente-Suarez I, Saha A, Cheroutre H, et al. Critical Role of Glucose Metabolism in Rheumatoid Arthritis Fibroblast-like Synoviocytes. Arthritis \& rheumatology (Hoboken, NJ). 2016;68(7):1614-26.

49. Lee MS, Moon EJ, Lee SW, Kim MS, Kim KW, Kim YJ. Angiogenic activity of pyruvic acid in in vivo and in vitro angiogenesis models. Cancer Res. 2001;61(8):3290-3.

50. Liang Q-h, He J-h, Li X-I. [Effect of bizhongxiao decoction on the expression of VEGF in the synovial membrane of C Il-induced rheumatoid arthritis in rats]. Hunan yi ke da xue xue bao = Hunan yike daxue xuebao = Bulletin of Hunan Medical University. 2002;27(6):491-4.

51. Sanchez-Lopez E, Cheng A, Guma M. Can Metabolic Pathways Be Therapeutic Targets in Rheumatoid Arthritis? J Clin Med. 2019;8(5).

52. Qian X, Yang Z, Mao E, Chen E. Regulation of fatty acid synthesis in immune cells. Scand J Immunol. 2018;88(5):e12713.

53. Ferreira HB, Melo T, Paiva A, Domingues MdR. Insights in the Role of Lipids, Oxidative Stress and Inflammation in Rheumatoid Arthritis Unveiled by New Trends in Lipidomic Investigations. Antioxidants (Basel). 2021;10(1).

54. Menendez JA, Lupu R. Fatty acid synthase (FASN) as a therapeutic target in breast cancer. Expert Opin Ther Targets. 2017;21(11):1001-16.

55. Berod L, Friedrich C, Nandan A, Freitag J, Hagemann S, Harmrolfs K, et al. De novo fatty acid synthesis controls the fate between regulatory $T$ and T helper 17 cells. Nat Med. 2014;20(11):1327-33.

56. Wei X, Song H, Yin L, Rizzo MG, Sidhu R, Covey DF, et al. Fatty acid synthesis configures the plasma membrane for inflammation in diabetes. Nature. 2016;539(7628):294-8.

57. Martín Al, Castillero E, Granado M, López-Menduiña M, Villanúa MA, López-Calderón A. Adipose tissue loss in adjuvant arthritis is associated with a decrease in lipogenesis, but not with an increase in lipolysis. J Endocrinol. 2008;197(1):111-9.

\section{Figures}

\section{Figure 1}

Page $15 / 20$ 
Effects of BZXD on the CIA rats. (A) Clinical scores index, (B) Paw thickness, (C) Body weight change was measured $0,7,14,21,28,35$ and 42 days ( $n=10$ rats per group, two-way repeated-measures ANOVA followed by Fisher's LSD test). (D) Serum inflammatory cytokines were measured 28 and 42 days by ELISA ( $n=4$ rats per group, one-way ANOVA followed by Fisher's LSD test). (E) Representative images of different degrees of arthritis in rats. Data are presented as mean $\pm \mathrm{SD}$, ${ }^{*} \mathrm{P}<0.05$, ${ }^{*} \mathrm{P}<0.01 \mathrm{CIA}$ versus Control; $\# \mathrm{P}<0.05 \# \# \mathrm{P}<0.01 \mathrm{BZXD}$ versus $\mathrm{CIA}$.

\section{Figure 2}

Histopathologic changes in the ankle joint of CIA rats affected by BZXD. Representative images of HE-stained (10X and $40 \mathrm{X})$ in the ankle joint of rats; scale bar represents $100 \mu \mathrm{m}(\mathrm{n}=5$ rats per group). Asterisks indicate inflammatory cells infiltration; arrows show synovium hyperplasia; Pentagrams show synovial neoangiogenesis; Triangles show bone destruction. 
A

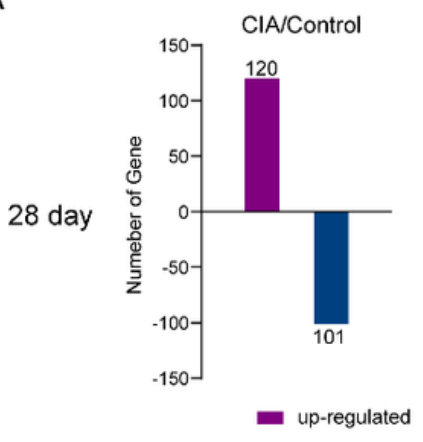

C

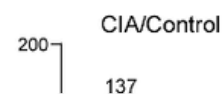

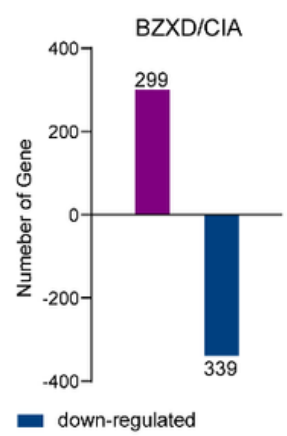

BZXD/CIA
B

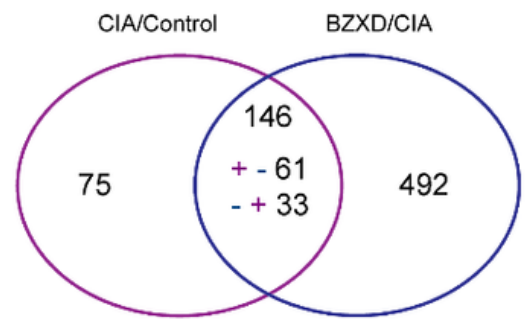

D

\section{Figure 3}

The differential proteins in CIA rats treated by BZXD. (A and C) The total number of identified DEPs from CIA/Control groups and BZXD/CIA groups on day 28 and 42. (B and D) Venn diagram of DEPs and their overlap. The "+" indicates up-regulated proteins, while the "-" indicates down-regulated proteins. The "+-" indicates proteins upregulated in $\mathrm{CIA}$ /Control groups but down-regulated in BZXD/CIA groups. The "-+" indicates proteins down-regulated in CIA/Control groups but up-regulated in BZXD/CIA groups. (E) Venn diagrams of the potential proteins associated with CIA and BZXD treatment on day 28 and 42. (F) The fold change dumbbell charts of potential proteins. Data were calculated by the Pearson correlation method after mean centering and unit variance scaling. 
A

The Most Enrichment GO Terms

negative regulation of JAK-STAT cascade hydrogen peroxide catabolic process response to hydrogen peroxide collagen fibril organization glutathione metabolic process oxygen transport

The Most Enrichment GO Terms 28d

B

The Most Enrichment GO Terms 42d protoporphyrinogen IX biosynthetic process oxidation-reduction process

integrin-mediated signaling pathway response to mercury ion heme biosvnthetic process

\section{Figure 4}

The functional classification of differentially expressed and reversely regulated by BZXD treatment proteins. (A and B) GO analysis of the 94 DEPs and 51 DEPs after being imported to DAVID on day 28 and 42 , respectively. ${ }^{*} p<0.05$

A

Pathway Enrichment
Valine, leucine and isoleucine degradation
Staphylococcus aureus infection.
Pyruvate metabolism.
Protein digestion and absorption.
Phagosome.
Pancreatic secretion.
Nitrogen metabolism.

B

Pathway Enrichment
Valine, leucine and isoleucine degradation
Synaptic vesicle cycle
Staphylococcus aureus infection
Pyruvate metabolism
Protein digestion and absorption
Phagosome
Pertussis

\section{Figure 5}

The pathways of DEPs of CIA/Control groups and BZXD/CIA groups on day 28 and 42. (A and B) The KEGG pathways enrichment analysis by DAVID. The color depth shows the -lg (FDR) values. Bubble size depicts the 
number of proteins identified in the pathway.

\section{Figure 6}

The PPI network of differentially expressed proteins. The PPI analysis of the 41 DEPs enriched in important pathways by STRING. Color depth represents the level of significance, with deeper colors indicating greater significance.

A
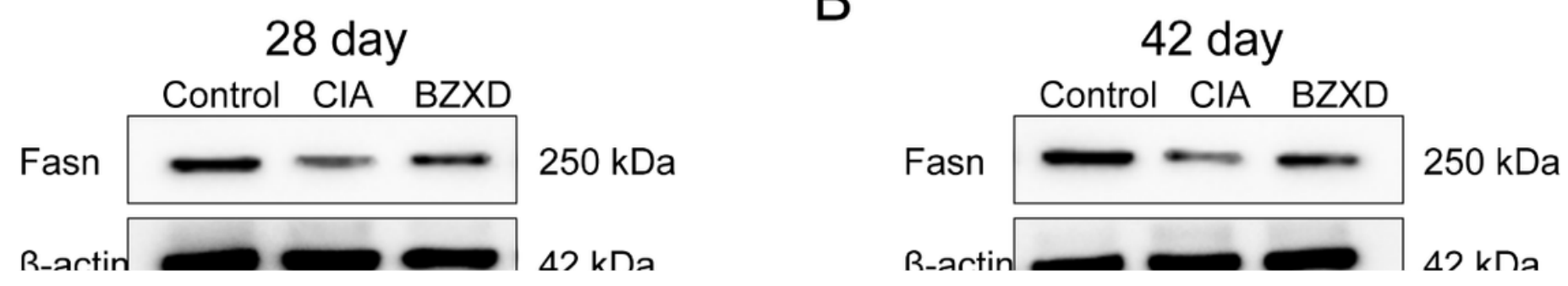

Figure 7

Effects of BZXD on the expression levels of FASN in CIA rats. Representative western blots of FASN (A and B) and their quantifications ( $C$ and D) among Control, CIA and BZXD groups on day 28 and 42. Data are presented as mean

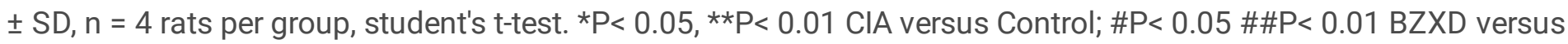
CIA. 


\section{Supplementary Files}

This is a list of supplementary files associated with this preprint. Click to download.

- SupplementaryFigure.pdf

- TableS1.docx

- Tables2.docx

- Tables3.docx 